\title{
Towards realistic SUSY grand unification for extended MSSM models
}

\author{
Sibo Zheng* \\ Department of Physics, Chongqing University, Chongqing 401331, People's Republic of China
}

(Received 12 September 2018; published 30 April 2019)

\begin{abstract}
Low-energy supersymmetric models such as the minimal supersymmetric standard model (MSSM), next-to-minimal supersymmetric standard model (NMSSM), and MSSM with vectorlike fermion are consistent with perturbative unification. While the nonminimal extensions naturally explain Higgs mass and dark matter in the low-energy region, it is unclear how seriously they are constrained in the ultraviolet region. Our study shows the following. First, in the case of embedding the MSSM into SU(5) the fit to standard model fermion masses requires a singlet $S$, which leads to unviable embedding of the NMSSM into SU(5) because such $S$ feeds singlet $N$ a mass of order unification scale as well. Second, a similar result holds in the case of embedding the NMSSM into $\mathrm{SO}(10)$, where $S$ is replaced by some Higgs fields responsible for $\mathrm{SO}(10)$ breaking. Third, on the contrary, for the embedding of the MSSM with 16-dimensional vectorlike fermions into SO(10), the Higgs field responsible for the vectorlike mass of order tera-electron-volts scale can evade those problems the singlet $N$ encounters because of an intermediate mass scale in the 126-dimensional Higgs field.
\end{abstract}

DOI: 10.1103/PhysRevD.99.075033

\section{INTRODUCTION}

At the frontiers of new physics, beyond the standard model (SM) natural or tera electron volt-scale supersymmetry (SUSY) offers us a grand unification (GUT) of SM gauge coupling constants [1-4]. Such natural SUSY hosts a lot of SUSY particles, which can be directly detected at the particle collider LHC or dark matter direct detection facilities such as Xenon-1T. Meanwhile, embedding these tera electron volt-scale SUSY models into the ultraviolet completions-SUSY GUT-may solve the long-standing issues such as the SM flavor puzzle and neutrino masses.

Nowadays, experimental data seem to oppose the minimal supersymmetric standard model (MSSM) from either the bottom or top viewpoint. In the former one, the $125 \mathrm{GeV}$ Higgs mass [5,6] requires either a large mixing effect or soft masses of order $10 \mathrm{TeV}$ for the stop scalars [7-9]. When the mixing effects among generations are significant, the constraints from flavor violation tend to require the SUSY mass order far above the weak scale. Moreover, the direct detection limits of dark matter $[10,11]$ impose rather strong pressure on the scenario of neutralino dark matter. In the latter perspective, the minimal SUSY SU(5) referring to the MSSM is significantly constrained

\footnotetext{
*sibozheng.zju@gmail.com
}

Published by the American Physical Society under the terms of the Creative Commons Attribution 4.0 International license. Further distribution of this work must maintain attribution to the author(s) and the published article's title, journal citation, and DOI. Funded by SCOAP ${ }^{3}$. by the proton decay [12]. It requires the color-triplet Higgs mass of order GUT scale, which together with unification leads to the MSSM mass spectrum at least of order $100 \mathrm{TeV}$ [13-15].

Therefore, it is of great interest to explore the MSSM with rational extensions that can resurrect the natural SUSY once again. Along this direction, there are at least two simple examples: the next-to-minimal supersymmetric standard model (NMSSM) [16] and the MSSM with vectorlike (VL) fermions (VMSSM) [17], which are consistent with unification $[18,19]$. While these extensions provide natural explanations of Higgs mass and dark matter in the low-energy region, it is unclear what the statuses of them are in the ultraviolet energy region. This is the main focus of this study.

In this paper, we discuss the embeddings of the MSSM, NMSSM and VMSSM into realistic GUT [20-22]. In each case, both the SUSY SU(5) [23-25] and SUSY SO(10) [26] representations will be explored. In these SUSY GUTs, we discuss the GUT-scale superpotential

$$
W=W_{Y}+W_{\mathrm{SB}},
$$

with the following features:

(i) $W_{Y}$ and $W_{\mathrm{SB}}$ are both renormalizable.

(ii) All of vacuum expectation values (vevs) are dynamically generated from $W_{\mathrm{SB}}$.

(iii) All of SM matters and extended matters obtain their masses via the Higgs mechanism in $W_{Y}$.

Since $W_{Y}$ is fixed by the SM and the extra matters such as the singlet $N$ or VL fermions at the tera-electron-volt 
scale, it is crucial to find suitable content of $W_{\mathrm{SB}}$ that achieves the breaking of gauge group $G_{\mathrm{GUT}} \rightarrow G_{\mathrm{SM}}$. In Sec. II, we explore the embedding of the MSSM into realistic SUSY GUTs, and useful conventions and notation will be introduced. The analysis on the MSSM is of great use to guide us towards the embeddings of the NMSSM and VMSSM. Sections III and IV are devoted to studying the embeddings of the NMSSM and VMSSM into realistic SUSY GUTs, respectively. Finally, we conclude in Sec. V.

\section{BENCHMARK MODEL: MSSM}

In the minimal SU(5), SM fermions of each generation are assigned as 1 for right-hand neutrino $N_{R}, \overline{5}(\psi)$ for $L$ and down quark $d$, and $10(\Phi)$ for $Q$, up quark $u$ and $e$, respectively, whereas in the $\mathrm{SO}(10)$ representation, the SM fermions of each generation are embedded into a 16dimensional representation, which decomposes as $16=$ $1+\overline{5}+10$ under the $\mathrm{SU}(5)$.

\section{A. SU(5)}

The Yukawa superpotential $W_{Y}$ in Eq. (1) contains two parts,

$$
W_{Y}^{\mathrm{SU}(5)}=W_{Y_{f}}^{\mathrm{SU}(5)}+W_{Y_{\nu}}^{\mathrm{SU}(5)},
$$

which refer to SM fermions without neutrinos and neutrinos, respectively.

According to the product $\overline{5} \times 10=5+45$ and $10 \times 10=\overline{5}_{s}+\overline{45}_{a}+5 \overline{50}_{s}$, where subscripts $\mathrm{s}$ and a refer to symmetric and antisymmetric, respectively. With the Higgs representations composed of 5, $\overline{5}$, and $\overline{45}$ [27], $W_{Y_{f}}^{\mathrm{SU}(5)}$ is given by

$$
\begin{aligned}
W_{Y_{f}}^{\mathrm{SU}(5)}= & Y_{d}^{i j} \psi_{a i}(\overline{5}) \bar{H}_{b}(\overline{5}) \Phi_{j}^{a b}(10) \\
& +Y_{45}^{i j} \psi_{a i}(\overline{5}) \bar{H}_{b c}^{a}(\overline{45}) \Phi_{j}^{b c}(10) \\
& +Y_{u}^{i j} \epsilon_{a b c d e} \Phi_{i}^{a b}(10) H^{e}(5) \Phi_{j}^{c d}(10)
\end{aligned}
$$

where $a, b, c$, etc., denote the $\mathrm{SU}(5)$ indices, $i$ and $j$ are the generation indices, and $Y_{u, d, 45}$ are Yukawa matrices. Note that $\Phi^{a b}$ is an antisymmetric field and $H_{b c}^{a}=-H_{c b}^{a}$.

The reason to include $\overline{45}$ is clear in the SM fermion mass matrices as derived from Eq. (3),

$$
\begin{aligned}
& M_{u}=Y_{u}^{i j} v_{u}^{5}, \\
& M_{d}=Y_{d}^{i j} v_{d}^{\overline{5}}+Y_{45}^{i j} v_{d}^{4 \overline{5}}, \\
& M_{e}=Y_{d}^{i j} v_{d}^{\overline{5}}-3 Y_{45}^{i j} v_{d}^{4 \overline{4}} .
\end{aligned}
$$

where $v_{u}^{5}$ and $v_{d}^{\overline{5}}$ is the vev of the Higgs doublet $H_{u}$ and $H_{d}$ in 5 and $\overline{5}$, respectively, and $v_{45}$ is the vev of doublet $\sigma_{d}$ in $\overline{45}$, which is defined as $\left\langle H_{a}^{b 5}\right\rangle=v_{45}(1,1,1,-3)_{\text {diag }}$ for $a, b=1-4$. Without the $\overline{45}, M_{d}=M_{e}$ at the GUT scale for all of three generations. While such a mass relation is viable for the third generation, it should be avoided for the first two generations. Adding $\overline{45}$ can tune the incorrect mass relations to the desired ones,

$$
m_{d} \simeq 3 m_{e}, \quad m_{s} \simeq m_{\mu} / 3, \quad m_{b} \simeq m_{\tau} .
$$

The GUT-scale mass relations in Eq. (5) strongly constrain the Yukawa matrices $Y_{u, d, 45}$. For example, some specific choices of $Y_{u, d, 45}$ in the Georgi-Jarlskog scheme [27] lead to a stable $b$ quark. To solve the SM flavor issue, we choose the Fritzsch scheme [28], in which $Y_{u, d, 45}$ take the forms

$$
\begin{aligned}
Y_{u}^{i j} v_{u} & =\left(\begin{array}{ccc}
0 & A_{u} & 0 \\
A_{u} & 0 & B_{u} \\
0 & B_{u} & C_{u}
\end{array}\right), \\
Y_{d}^{i j} v_{d} & =\left(\begin{array}{ccc}
0 & A_{d} & 0 \\
A_{d} & 0 & B_{d} \\
0 & B_{d} & C_{d}
\end{array}\right), \\
Y_{45}^{i j} v_{45} & =\left(\begin{array}{ccc}
0 & 0 & 0 \\
0 & 0 & D_{d} \\
0 & D_{d} & 0
\end{array}\right),
\end{aligned}
$$

where there are small mass hierarchies $C_{f} \gg B_{f} \gg A_{f}$ with $f=\{u, d, e\}$ in Eq. (6) so as to address the SM flavor mass hierarchies. Substituting Eq. (6) into Eq. (5) implies that there is a fine-tuning between $B_{d}$ and $D_{d}$,

$$
D_{d}=\left(1 \pm \frac{2 \sqrt{3}}{3}\right) B_{d}
$$

With this fine-tuning solution, the diagonalizations of matrices in Eq. (6) yield the Cabibbo-KobayashiMaskawa (CKM) matrix

$$
\left|V_{\mathrm{CKM}}\right| \simeq\left(\begin{array}{lll}
0.974 & 0.227 & 0.004 \\
0.227 & 0.970 & 0.042 \\
0.008 & 0.042 & 0.999
\end{array}\right),
$$

which is in good agreement with experimental data.

For $W_{Y_{\nu}}$ responsible neutrino masses, we take a simple form,

$$
\begin{aligned}
W_{Y_{\nu}}^{\mathrm{SU}(5)}= & Y_{N}^{i j} N_{R i}(1) \psi_{a j}(\overline{5}) H^{a}(5) \\
& +Y_{S}^{i j} S(1) \cdot N_{R i}(1) N_{R j}(1)+\text { H.c., }
\end{aligned}
$$

where $S$ is a singlet of $G_{\mathrm{SM}}=S U(3)_{c} \times S U(2)_{L} \times U(1)_{Y}$, with $\langle S\rangle$ of order GUT scale. In Eq. (9), one finds the neutrino Dirac mass $M_{\nu}=Y_{N}^{i j} v_{u}^{5}$ and the right-handed 
neutrino mass $M_{N_{R}}=Y_{S}^{i j}\langle S\rangle$, which results in the left-hand neutrino masses in terms of the type-I [29-32] seesaw mechanism,

$$
m_{\nu}=M_{\nu}^{T} M_{N_{R}}^{-1} M_{\nu}=Y_{N}^{T} Y_{S}^{-1} Y_{N} \frac{v_{u}^{5}}{\langle S\rangle} .
$$

Given $v_{u}^{5} \sim 10^{2} \mathrm{GeV},\langle S\rangle \sim 10^{15}-10^{16} \mathrm{GeV}$, and Yukawa couplings of order unity, the neutrino mass is of order approximately $10^{-2}-10^{-3} \mathrm{eV}$. Similar to $Y_{u, d, e}, Y_{S}$ and $Y_{N}$ in Eq. (9) are also constrained by the fit to neutrino mixings as described by the Pontecorvo-Maki-NakagawaSakata (PMNS) matrix $U_{\mathrm{PMNS}}=U_{e}^{-1} U_{\nu}^{-1}$, where $U_{\nu}$ and $U_{e}$ are defined to diagonalize mass matrices $m_{\nu}$ and $M_{e}$, respectively.

Now that we have established a benchmark solution ${ }^{1}$ to the input parameters at the GUT scale that can explain the SM flavor issue and neutrino masses, we turn to the structure of $\left.W_{\mathrm{SB}}: 1\right)$ To obtain light neutrino masses, $\langle S\rangle$ of order GUT scale is required. 2). To break the SU(5), we introduce $75(Z)$. With a 75 , we can add a 50 and 50 to
TABLE I. $\quad Z_{2} \times Z_{2}^{\prime}$ parity assignments in the case of embedding the MSSM into SU(5), which are consistent with the superpotentials in Eqs. (2) and (12).

\begin{tabular}{lllllllllllllll}
\hline \hline Field & $N_{R}$ & $\psi$ & $\Phi$ & 5 & $\overline{5}$ & $\overline{45}$ & $1(S)$ & $1\left(S^{\prime}\right)$ & $75(Z)$ & $75\left(Z^{\prime}\right)$ & 45 & 50 & 50 \\
\hline$Z_{2}$ & + & + & - & + & - & - & + & + & + & - & - & - & - \\
$Z_{2}^{\prime}$ & + & + & + & + & + & + & + & - & + & - & + & + & - \\
\hline \hline
\end{tabular}

achieve the doublet-triplet splitting [33] for 5 and $\overline{5}$. 3). To gain nonzero vev $v_{d}^{\overline{45}}$, we include another $75\left(Z^{\prime}\right)$ with a vev of the GUT scale. The reason for this is that neither 1 nor 24 with a large vev is favored by the product $H(5) \times \bar{H}(\overline{5})=$ $1+24$. 4), Because of the singlet $S$, there is an unsafe operator,

$$
W_{\text {unsafe }}=S H(5) \bar{H}(\overline{5}),
$$

which must be eliminated.

Shown in Table $\mathrm{I}$ is the $Z_{2} \times Z_{2}^{\prime}$ parity, which can eliminate the unsafe operator in Eq. (11). Under this parity, $W_{\mathrm{SB}}$ reads as

$$
\begin{aligned}
W_{\mathrm{SB}}^{\mathrm{SU}(5)}= & \frac{M_{Z}}{2} Z^{2}+\frac{M_{Z^{\prime}}}{2} Z^{\prime 2}+\frac{M_{S}}{2} S^{2}+\frac{M_{S^{\prime}}}{2} S^{\prime 2}+\frac{\lambda_{Z}}{3} Z^{3}+\frac{\lambda_{S}}{3} S^{3}+\lambda_{1} S Z^{2}+\lambda_{2} S Z^{\prime 2}+\lambda_{3} Z Z^{\prime 2}+\lambda_{4} S S^{\prime 2} \\
& +\bar{H}(\overline{45})\left(M_{45}+\lambda_{5} S\right) H(45)+\lambda_{6} S^{\prime} \bar{H}(\overline{50}) H(50)+\lambda_{7} Z \bar{H}(\overline{5}) H(50)+\lambda_{8} Z^{\prime} H(5) \bar{H}(\overline{50})+\lambda_{9} Z H(45) \bar{H}(\overline{5})+\text { H.c. }
\end{aligned}
$$

According to the $F$ terms in Eq. (12), the nonzero singlet vevs $\left\langle(1,1,1)_{Z}\right\rangle=Z,\left\langle(1,1,1)_{Z^{\prime}}\right\rangle=Z^{\prime},\left\langle(1,1,1)_{S}\right\rangle=S$ and $\left\langle(1,1,1)_{S^{\prime}}\right\rangle=S^{\prime}$ are given by

$$
\begin{aligned}
\frac{S}{M_{Z}} & =-\frac{1}{2 \lambda_{4}} \eta_{3}=-\frac{1}{2 \lambda_{2}}\left(-2 \lambda_{3} \frac{Z}{M_{Z}}+\eta_{1}\right), \\
\frac{Z^{\prime}}{M_{Z}} & =\frac{1}{\sqrt{\lambda_{S}}}\left(\frac{-Z}{M_{Z}}\right)^{\frac{1}{2}}\left[1+\lambda_{Z}+\frac{\lambda_{1}}{\lambda_{2}}\left(-2 \lambda_{3} \frac{Z}{M_{Z}}+\eta_{1}\right)\right]^{\frac{1}{2}} \\
\frac{Z}{M_{Z}} & =\frac{-b \pm \sqrt{b^{2}-4 a c}}{2 a},
\end{aligned}
$$

where

$$
\begin{aligned}
& a=\lambda_{1}+\frac{\lambda_{3}^{2}}{\lambda_{2}^{2}} \lambda_{S}-\frac{\lambda_{2} \lambda_{Z}}{\lambda_{S}}+2 \frac{\lambda_{1} \lambda_{3}}{\lambda_{S}} \\
& b=-\frac{\lambda_{3}}{\lambda_{2}}-\frac{\lambda_{3} \lambda_{S}}{\lambda_{2}^{2}} \eta-\frac{\lambda_{2}}{\lambda_{S}}-\frac{\lambda_{1}}{\lambda_{S}} \eta \\
& c=\frac{1}{2 \lambda_{2}} \eta_{1} \eta_{2}+\frac{\lambda_{S}}{4 \lambda_{2}^{2}} \eta_{1}^{2}+\lambda_{4}\left(\frac{S^{\prime}}{M_{Z}}\right)^{2}
\end{aligned}
$$

\footnotetext{
${ }^{1}$ It is of special interest to examine whether there is a viable solution to the input parameters in the case in which all mass matrices such as $M_{u, d, e, N_{R}}$ are assigned the Fritzsch form.
}

and $\eta_{1}=M_{Z^{\prime}} / M_{Z}, \eta_{2}=M_{S} / M_{Z}, \eta_{3}=M_{S^{\prime}} / M_{Z}$.

A few comments are in order regarding the parity assignments. First, the $Z_{2}$ parity eliminates the unsafe operator in Eq. (11). Second, without $Z_{2}^{\prime}$, a large $\mu$ term for the doublets in 5 and $\overline{5}$ would be induced by the mixings with 45 and $\overline{45}$. Instead, imposing $Z_{2}^{\prime}$ forbids the operator $Z^{\prime} H(5) \bar{H}(\overline{45})$, which then keeps the doublets in 5 and $\overline{5}$ light. Finally, due to the last line in Eq. (12), which is consistent with $Z_{2} \times Z_{2}^{\prime}$, the effective operator $\bar{H}(\overline{5}) H(45) Z^{\prime 2} / M_{Z}$ is produced after integrating $Z$. Thus, the effective superpotential for the doublets in 45 and $\overline{45}$ at the leading order is given by

$$
W_{\text {eff }} \sim\left(M_{45}+\lambda_{5}\langle S\rangle\right) \sigma_{u} \sigma_{d}+\lambda_{3} H_{d} \sigma_{u} Z^{\prime 2} / M_{Z}
$$

where corrections due to those mixings among singlets of $Z, Z^{\prime}$, and $S$ have been neglected. From Eq. (15), we obtain the vev

$$
\sigma_{d} \sim \lambda_{3} \frac{Z^{\prime 2}}{\left(M_{45}+\lambda_{4}\langle S\rangle\right) M_{Z}} v_{d}
$$

Given $\lambda_{3} \sim 0.1$ and $v_{d} \sim 10 \mathrm{GeV}$, we have $\sigma_{d} \sim 100 \mathrm{MeV}$. A rational Yukawa texture such as $Y_{45} \sim(0.01,0.1,1)$ for 
the three generations then reproduces the mass relations in Eq. (5).

\section{B. $\operatorname{SO}(10)$}

Unlike the case of $\mathrm{SU}(5)$, the input parameters in the $\mathrm{SO}(10)$ that control the SM fermions masses $M_{u, d, e}$ and neutrino masses $m_{\nu}$ are tied to each other. The main reason for this is that the MSSM matters of each generation are contained in a single $16(\phi)$. Here, we give a brief review of the embedding of the MSSM into $\mathrm{SO}(10)$.

According to the product $16 \times 16=10_{s}+120_{a}+126_{s}$, the Higgs representation responsible for SM fermion masses can be composed of 10,120 , and $1 \overline{2} 6$. By following previous discussions on $\mathrm{SU}(5)[34,35]$, the simplest choice is to introduce 10 and $1 \overline{2} 6$ [36], with Yukawa superpotential given by

$W_{Y}^{\mathrm{SO}(10)}=\phi_{i}(16)\left[Y_{10}^{i j} H(10)+Y_{126}^{i j} \bar{H}(1 \overline{2} 6)\right] \phi_{j}(16)$,

where matrices $Y_{10}$ and $Y_{126}$ are both symmetric. From Eq. (17), we have

$$
\begin{aligned}
& M_{u}=Y_{10}^{i j} v_{u}^{10}+Y_{126}^{i j} v_{u}^{12 \overline{6}}, \\
& M_{d}=Y_{10}^{i j} v_{d}^{10}+Y_{126}^{i j} v_{d}^{12 \overline{6}}, \\
& M_{e}=Y_{10}^{i j} v_{d}^{10}-3 Y_{126}^{i j} v_{d}^{1 \overline{2} 6}
\end{aligned}
$$

for SM quarks and electrons and

$$
\begin{aligned}
M_{N_{R}} & =Y_{126}^{i j} v_{s}^{1 \overline{2} 6} \\
M_{\nu} & =Y_{10}^{i j} v_{u}^{10}-3 Y_{126}^{i j} v_{d}^{1 \overline{12} 6}, \\
M_{L} & =Y_{126}^{i j} v_{L}^{1 \overline{2} 6}
\end{aligned}
$$

for neutrinos, where $v_{u, d}^{10}$ refer to the doublet vevs in $H(10)$ and $v_{s, u, d, L}^{1 \overline{2}}$ denote the singlet (s), doublet (u, d), and triplet (L) vevs in $\bar{H}(1 \overline{2} 6)$, respectively. Therefore, the neutrino mass arises from both type-I [29-32] and type-II [37-39] contributions,

$$
m_{\nu}=M_{L}-M_{\nu}^{T} M_{N_{R}}^{-1} M_{\nu}
$$

Benchmark solutions to the input parameters in Eqs. (18) and (19) have been extensively studied [36,40-43], which demonstrates that the fit to SM flavor masses and mixings is viable. But the construction of $W_{\mathrm{SB}}$ responsible for the breaking of $\mathrm{SO}(10) \rightarrow G_{\mathrm{SM}}$ is challenging [44-47], since some of the triplet fields in 126 and $1 \overline{2} 6$ obtain mass of order $v_{s}^{1 \overline{2} 6} \sim 10^{13} \mathrm{GeV}$, which spoils the perturbative unification. Attempts to solve this issue involve adding 54 [48] to $W_{\mathrm{SB}}$ or adding 120 [49] to $W_{Y}$ in Eq. (17).
We employ the solution of modifying $W_{\mathrm{SB}}$ [48], in which the Higgs fields are composed of $210(Y), H(126), \bar{H}(1 \overline{2} 6)$, and $54(X)$, and $W_{\mathrm{SB}}$ takes the form ${ }^{2}$

$$
\begin{aligned}
W_{\mathrm{SB}}^{\mathrm{SO}(10)}= & \frac{M_{Y}}{2} Y^{2}+\frac{M_{X}}{2} X^{2}+M_{126} H(126) \bar{H}(1 \overline{2} 6) \\
& +\lambda_{1} Y^{3}+\lambda_{2} Y H(126) \bar{H}(1 \overline{2} 6) \\
& +Y\left[\lambda_{3} H(126) H(10)+\lambda_{4} \bar{H}(1 \overline{2} 6) H(10)\right] \\
& +X\left[\lambda_{8} X^{2}+\lambda_{10} Y^{2}+\lambda_{11} H^{2}(126)\right. \\
& \left.+\lambda_{12} \bar{H}^{2}(1 \overline{2} 6)+\lambda_{13} H^{2}(10)\right]+ \text { H.c. }
\end{aligned}
$$

Here, a few comments about $W_{\mathrm{SB}}$ are in order: 1). Under the notation of $S U(4) \times S U(2)_{L} \times S U(2)_{R}$, the $S M$ singlet vevs $Y_{1}=\left\langle(1,1,1)_{Y}\right\rangle, \quad Y_{2}=\left\langle(15,1,1)_{Y}\right\rangle, \quad$ and $Y_{3}=$ $\left\langle(15,1,3)_{Y}\right\rangle$ in $Y$ and $X=\left\langle(1,1,1)_{X}\right\rangle$ in $X$ are responsible for the breaking $\mathrm{SO}(10) \rightarrow G_{\mathrm{SM}} \times U(1)_{B-L}$. 2). The $\mathrm{SM}$ singlet vevs $v_{s}^{126}=\left\langle(\overline{10}, 1,3)_{126}\right\rangle$ and $v_{s}^{1 \overline{2} 6}=\left\langle(10,1,3)_{1 \overline{2} 6}\right\rangle$ result in the breaking of $G_{\mathrm{SM}} \times U(1)_{B-L} \rightarrow G_{\mathrm{SM}}$.

In the limit $v_{s}^{126}=v_{s}^{126} \ll Y_{i}, X$ the vevs are given by [48]

$$
\begin{aligned}
\frac{Y_{1}}{Y_{2}} & =\frac{1}{2 \sqrt{3}} \eta^{2}, \\
\frac{M_{Y}}{Y_{2}} & =-\frac{\lambda_{1}}{5 \sqrt{2}}\left(3+\eta^{2}\right), \\
\frac{X}{Y_{2}} & =\frac{\lambda_{1}}{\lambda_{10} \sqrt{30}}\left(-2+\eta^{2}\right), \\
\frac{M_{126}}{Y_{2}} & =-\frac{\lambda_{2}}{120}\left(6 \sqrt{2}+12 \eta+\sqrt{2} \eta^{2}\right), \\
\frac{M_{X}}{Y_{2}} & =\frac{-1}{20 \sqrt{2} \lambda_{1} \lambda_{10}}\left[\left(5 \lambda_{10}^{3}+2 \lambda_{1}^{2} \lambda_{8}\right) \eta^{2}+20 \lambda_{10}^{3}-4 \lambda_{1}^{2} \lambda_{8}\right]
\end{aligned}
$$

with $\eta=Y_{3} / Y_{2}$.

\section{NMSSM}

With the embedding of the MSSM into realistic SUSY GUTs as a benchmark, in this section, we analyze the NMSSM. According to the starting points in the Introduction, a viable embedding should satisfy two constraints:

(i) The mass of $N$ should be of order tera-electronvolt scale.

(ii) The vev of $N$ should be of order tera-electronvolt scale.

Both of them may be spoiled by a few dangerous mixings between $N$ and Higgs fields that contain a singlet vev of order GUT scale. The key point is whether there is suitable symmetry to avoid such mixings.

\footnotetext{
${ }^{2}$ We follow the notation in Ref. [50] for Yukawa coupling constants.
} 


\section{A. $\mathbf{S U}(5)$}

In this situation, $W_{Y}$ in Eq. (2) should be extended to include $\mathrm{NH}_{u} \mathrm{H}_{d}+\frac{\kappa}{3} \mathrm{~N}^{3}+$ H.c. in the NNSSM, which means that ${ }^{3}$

$$
\delta W_{Y}=\lambda N H(5) \bar{H}(\overline{5})+\frac{\kappa}{3} N^{3} .
$$

Equation (23) does not affect the fit to SM flavor masses and mixings in Sec. II A. Nevertheless, compared to the MSSM, $W_{\mathrm{SB}}$ is allowed to contain superpotential terms

$$
W_{\text {unsafe }}=N Z^{2}+N Z^{\prime 2}+N S^{2}+N^{2} S .
$$

These new terms in Eq. (24) yield corrections to the $F$ terms of $Z, Z^{\prime}$, and $S$ such as $F_{S}=F_{S}^{\mathrm{MSSM}}+2 \lambda_{N} S N$, which can be adjusted to the case of the MSSM by, e.g., $\langle N\rangle=0$. Even so, the singlet vevs $\langle Z\rangle,\left\langle Z^{\prime}\right\rangle$, and $\langle S\rangle$ still lead to either large $N$ mass or large mixing.

To avoid all of the mixing terms in Eq. (24), we need to impose new parity. The first observation is that an odd $N$ under a $Z_{2}$ parity as shown in Table I excludes the first three terms in Eq. (24). But the last term therein still remains. ${ }^{4}$ A similar result holds for $Z_{N}$ or an Abelian symmetry.

\section{B. $\operatorname{SO}(10)$}

Similar to the embedding of the NMSSM into SU(5), $W_{Y}$ in Eq. (17) is modified by

$$
\delta W_{Y}=\lambda N H^{2}(10)+\frac{\kappa}{3} N^{3} .
$$

Instead of Eq. (24), $W_{\mathrm{SB}}$ in Eq. (21) is allowed by gauge invariance to contain

$$
W_{\text {unsafe }}=N Y^{2}+N H(126) \bar{H}(1 \overline{2} 6)+N X^{2}+X H^{2}(10),
$$

in which $N$ mixes with the SM singlets of $Y, 126,1 \overline{2} 6$, and $X$. Thus, all of Yukawa couplings in Eq. (27) have to be extremely small.

What kind of parity allows Yukawa superpotential in Eq. (26) but eliminates that in Eq. (27) simultaneously? The first observation is that a $Z_{2}$ parity does not work, since

\footnotetext{
${ }^{3}$ Operator $N H(5) \bar{H}(\overline{5})$ contributes to Yukawa interaction $N H_{c} \bar{H}_{c}$ beyond the MSSM, with $H_{c}$ and $\bar{H}_{c}$ being the colortriplet Higgs fields. However, it does not affect proton decay at all, as the singlet $N$ mass is always far larger than proton mass.

${ }^{4}$ An economic solution to keeping light $N$ is adding another singlet $S^{\prime}=1$ that is even under the $Z_{2}$. With such an $S^{\prime}$, the $W_{\mathrm{SB}}$ is further extended by$$
\delta W_{\mathrm{SB}}\left(S^{\prime}\right)=\frac{1}{2} M_{S^{\prime}} S^{\prime 2}+m_{S S^{\prime}} S S^{\prime}+N^{2} S^{\prime}
$$

from which $F_{S^{\prime}}=M_{S^{\prime}} S^{\prime}+m_{S S^{\prime}} S+N^{2}$, and $\left\langle S^{\prime}\right\rangle=-\langle S\rangle$ if $M_{S^{\prime}}=m_{S S^{\prime}}$. The two different contributions to the $N$ mass cancel each other, leaving us a light $N$. Unfortunately, neither Abelian or $Z_{N}$ parity can ensure $M_{S^{\prime}}=m_{S S^{\prime}}$.
}

Eq. (27) would imply that $N$ is an odd field, which contradicts with the Yukawa superpotential in Eq. (26). Similar results hold for any $Z_{N}$ parity. Because the rational assignment $n_{Y}=0$ as required by successful symmetry breaking implies that $n_{1} 0=n_{1} 26=n_{12} 6=N / 2$ in order to allow the Yukawa superpotential in Eq. (26). Accordingly, $n_{N}=0$ from $N 10(H) 10(H)$, which implies that some of the terms in Eq. (27) are still allowed. To conclude, in our setup, embedding the NMSSM into the minimal $\mathrm{SO}(10)$ is not viable.

\section{VMSSM}

Let us proceed to discuss the embedding of the VMSSM into SUSY SU(5) and SO(10). The VL fermions with mass of order $\mathrm{TeV}$ scale can be composed of 5 with $\overline{5}, 10$ with $\overline{10}$ in the $\mathrm{SU}(5)$, or 16 with $\overline{16}$ in the $\mathrm{SO}(10)[18,19]$. A realistic embedding should satisfy the following constraints:

(i) The vev of the Higgs field $\rho$ responsible for the VL fermion masses should be of order tera-electronvolt scale.

(ii) The VL fermions are prevented from directly coupling to the Higgs fields which trigger high-scale gauge symmetry breaking.

Violating the first constraint is likely to occur because either $\rho=\{1,24,75\}$ or $\rho=\{1,45,210\}$ may directly couple to $S, S^{\prime} Z, Z^{\prime}$ in the case of $\mathrm{SU}(5)$ or $X$, and $Y$ in the case of $\mathrm{SO}(10)$, which tends to yield $\langle\rho\rangle$ of GUT scale. In contrast, $\langle\rho\rangle$ of order tera-electron-volt scale can be only realized by the effective operator such as

$$
W_{\text {eff }}(\rho)=\frac{M_{\rho}}{2} \rho^{2}+\frac{\rho \cdot A \cdot B \cdot \cdots}{M_{\mathrm{U}}^{n}},
$$

where $A, B, \cdots$, refer to $H(5), \bar{H}(\overline{5}), S, S^{\prime}, Z$, and $Z^{\prime}$ in the $\mathrm{SU}(5)$ or $H(126), \bar{H}(1 \overline{2} 6), X$, and $Y$ in the $\mathrm{SO}(10)$, with $M_{U}$ denoting the GUT scale.

\section{A. $\mathbf{S U}(\mathbf{5})$}

For the VL fermions of $5(\Sigma)$ and $\overline{5}(\bar{\Sigma}), W_{Y}$ in Eq. (2) is extended by

$$
\delta W_{Y}=\rho \bar{\Sigma}(\overline{5}) \Sigma(5)+\text { H.c. }
$$

where $\rho=\{1,24\}$ of $\mathrm{SU}(5)$. The reason for adding $\rho$ is that either singlet vev $S$ or $S^{\prime}$ in Sec. II A is too large to provide a VL mass of order tera-electron-volts.

TABLE II. $\quad Z_{2}$ parity assignments for embedding the NMSSM into $\mathrm{SU}(5)$, which is consistent with the superpotential in Eq. (29). Yet, This parity is unable to exclude all the unsafe structures in Eq. (31).

Field $N_{R} \psi \Phi \Sigma \bar{\Sigma} 5 \overline{5} \overline{45} \rho 1(S) 1\left(S^{\prime}\right) 75(Z) 75\left(Z^{\prime}\right) 45505 \overline{0}$ Z $Z_{2}++-+-+---++\quad+\quad+\quad-\quad-\quad-$ 
In this case, the unsafe superpotential at least includes

$$
W_{\text {unsafe }} \supset S \Sigma \bar{\Sigma},
$$

which can be excluded by imposing the $Z_{2}$ parity assignments as shown in Table II. $W_{\text {unsafe }}$ in Eq. (30) can also contain the following terms depending on $\rho$ :

$$
W_{\text {unsafe }}= \begin{cases}\rho Z^{2}+\rho Z^{\prime 2}+S \rho Z^{\prime}+\rho^{2} S, & \rho=24 \\ \rho Z^{2}+\rho Z^{\prime 2}+\rho^{2} S+\rho S^{2}, & \rho=1 .\end{cases}
$$

Besides the unsafe operators in Eq. (31), there are also no suitable Feynman graphs to generate the desired effective operator with correct mass order in Eq. (28). In principle, the form of effective operator in Eq. (28) can be divided as follows:

(i) It is of type $\rho A B \cdots / M_{\mathrm{U}}^{2}$, with $A$ and $B$ referring to $S, Z$, or $Z^{\prime}$. In this situation, one obtains $\langle\rho\rangle \sim$ GUT scale, given all of $A, B, \cdots$, are of order GUT scale.

(ii) It looks like $\rho A B \cdots H(5) / M_{\mathrm{U}}$ or $\rho A B \cdots \bar{H}(\overline{5}) /$ $M_{\mathrm{U}}$, which contains only a doublet vev. Such an operator contributes to $\langle\rho\rangle \sim \mathrm{TeV}$ for the $M_{\rho} \sim \mathrm{GUT}$ scale. Unfortunately, there is no suitable intermediator.

(iii) It is of the form $\rho A B \cdots H(5) \bar{H}(\overline{5}) \cdots / M_{\mathrm{U}}^{2}$ or $\rho A B \cdots \bar{H}(\overline{5}) H(45) \cdots / M_{\mathrm{U}}^{2}$, where there are at least two doublet vevs. In this case, $\langle\rho\rangle$ is less than $\mathcal{O}(1) \mathrm{eV}$.

In summary, since there is no appropriate vev scale, the embedding of the VMSSM into $\mathrm{SU}(5)$ is not viable. Similar result can be found for VL 10 and $\overline{10}$, where $\rho=$ $\{1,24,75\}$ of $\mathrm{SU}(5)$.

\section{B. $\operatorname{SO}(10)$}

As shown in Ref. [19], the nonminimal extension through the 16-dimensional VL fermions remains consistent with the $\mathrm{SO}(10)$ unification. In this model, $W_{Y}$ is modified by

$$
\delta W_{Y}=\rho \Delta(16) \bar{\Delta}(\overline{16}),
$$

where $\rho=\{1,45,210\}$ of $\mathrm{SO}(10)$. Similar to Sec. IVA, the main challenge to the embedding is the generation of singlet vev $\langle\rho\rangle \sim \mathrm{TeV}$ scale for a $\rho$ mass of GUT scale. What differs from the discussions in Sec. IVA is the existence of the intermediate mass scales $v_{s}^{126}$ and $v_{s}^{1 \overline{2} 6}$ in the $\mathrm{SO}(10)$, which is critical to solving the problem.

TABLE III. $Z_{2}$ parity assignments in the VMSSM model with $\rho=210$, which are consistent with the superpotentials in Eqs. (17), (21), (32), and (34) and simultaneously avoid the unsafe operator in Eq. (33).

\begin{tabular}{lccccccccccc}
\hline \hline Field & $\phi$ & $\Delta$ & $\bar{\Delta}$ & 10 & $1 \overline{2} 6$ & $\rho$ & $X$ & 126 & $54(V)$ & $54(U)$ & $Y$ \\
\hline$Z_{2}$ & + & + & - & + & + & - & - & + & - & - & + \\
\hline \hline
\end{tabular}

We first consider $\rho=210$. We add Higgs fields 54(V) and $54(U)$ to $W_{\mathrm{SB}}$, with the $Z_{2}$ parity assignments as shown in Table III. The $Z_{2}$ parity excludes the unsafe operator

$$
W_{\text {unsafe }}=Y \Delta(16) \bar{\Delta}(\overline{16})
$$

and simultaneously allows Yukawa interactions

$$
\begin{aligned}
\delta W_{\mathrm{SB}}= & \frac{M_{\rho}}{2} \rho^{2}+\frac{M_{V}}{2} V^{2}+\frac{M_{U}}{2} U^{2} \\
& +\lambda_{10}^{\prime} \rho U Y+\lambda_{10}^{\prime \prime} \rho V Y \\
& +\lambda_{8}^{\prime} U V X+\lambda_{8}^{\prime \prime} U^{2} X+\lambda_{8}^{\prime \prime \prime} V^{2} X .
\end{aligned}
$$

In terms of Eqs. (34) and (21), the effective operator for $\rho$ is given by

$$
\begin{aligned}
W_{\text {eff }} \simeq & \frac{M_{\rho}}{2} \rho^{2}+\rho\left(\lambda_{10}^{\prime} U+\lambda_{10}^{\prime \prime} V\right)\left[\frac{H(126) \bar{H}(1 \overline{2} 6)}{M_{Y}}+\cdots\right] \\
& +\rho \frac{\lambda_{10}^{\prime} U H^{3}(126) \bar{H}(1 \overline{2} 6)}{M_{Y} M_{X} M_{V}}+\rho \frac{\lambda_{10}^{\prime \prime} V H^{3}(126) \bar{H}(1 \overline{2} 6)}{M_{Y} M_{X} M_{U}} \\
& +\mathcal{O}\left(\rho^{3}\right) .
\end{aligned}
$$

For calculating an effective superpotential in the infrared region from those in the ultraviolet region, integrating out heavy chiral superfields in the Feynman graphs is equivalent to solving the nonlinear equations of $F$ terms related to these heavy chiral superfields. The leading-order terms with coefficient $\lambda_{10}^{\prime} U+\lambda_{10}^{\prime \prime} V=\delta F_{Y}$ in Eq. (35) are obtained after integrating out superfield $Y$ for $\lambda_{1}$ and $\lambda_{10}$ less than unity. Similarly, the next-leading-order operators therein are induced by further integrating out $X$, referring to which the Feynman graph is shown in Fig. 1. Note that we have used the mass term in Eq. (35) representing those quadratic terms and neglected the higher-order terms.

Apart from the $F$-term contributions in Eq. (35), the potential for the singlet component in $\rho$ also contains soft SUSY-breaking terms such as $A_{\rho} \rho^{3}+$ H.c. The scale of $A_{\rho}$ depends on the details of SUSY breaking. It can be neglected in gauge mediation but be of order approximately $1 \mathrm{TeV}$ in the other scenarios. In the latter case, it is easily to verify that the $F$-term contribution still dominates over

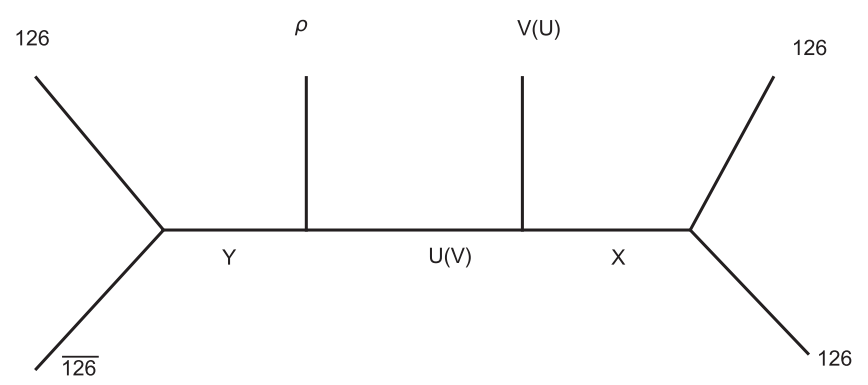

FIG. 1. Super-Feynman graph for the generation of the higherdimensional effective operator in the case of $\rho=210$. 
those soft terms. In what follows, we simply ignore those soft terms for the estimate of $\langle\rho\rangle$.

The leading-order contribution in Eq. (35) has to be eliminated if one wants to obtain singlet vev $\langle\rho\rangle \sim \mathrm{TeV}$ scale. Alternatively, the coefficient $\delta F_{Y}$ must be suppressed without much fine-tuning. There is a dynamical realization for this purpose. From Eq. (34), one finds that the SUSY vacuum described by the vevs in Eq. (22) remains only if the constraints

$$
\begin{aligned}
& 0=\lambda_{8}^{\prime} U V+\lambda_{8}^{\prime \prime} U^{2}+\lambda_{8}^{\prime \prime \prime} V^{2}, \\
& 0=\lambda_{10}^{\prime} U+\lambda_{10}^{\prime \prime} V, \\
& 0=M_{V} V+\frac{X}{2 \sqrt{15}}\left(\lambda_{8}^{\prime} U+2 \lambda_{8}^{\prime \prime \prime} V\right), \\
& 0=M_{U} U+\frac{X}{2 \sqrt{15}}\left(\lambda_{8}^{\prime} V+2 \lambda_{8}^{\prime \prime} U\right)
\end{aligned}
$$

are satisfied. Given GUT-scale mass parameters $M_{U}, M_{V}$, $\langle U\rangle$, and $\langle V\rangle$, there are indeed rational solutions to Eq. (36), under which the leading-order operator in Eq. (35) vanishes due to $\delta F_{Y}=0$ [the second formula in Eq. (36)]. Therefore, the next-leading-order contribution in Eq. (35) dominates the effective superpotential for $\rho$ below GUT scale as long as $M_{U} \neq M_{V}$,

$$
W_{\text {eff }} \sim M_{\rho} \rho^{2}+\rho \frac{V H^{3}(126) \bar{H}(1 \overline{2} 6)}{M_{U}^{3}},
$$

which contributes to a nonzero vev:

$$
\langle\rho\rangle \sim \frac{\langle V\rangle}{M_{\rho}} \frac{\left(v_{s}^{126}\right)^{4}}{M_{U}^{3}} .
$$

Given singlet vev $v_{s}^{126} \simeq v_{s}^{1 \overline{1} 6} \sim 10^{13} \mathrm{GeV}$ fixed by the fit to SM flavor masses [48] and $M_{\rho} \sim M_{U} \sim\langle V\rangle \sim 10^{16} \mathrm{GeV}$, we have $\langle\rho\rangle \sim 1-10 \mathrm{TeV}$.

The analysis for $\rho=210$ can shed light on other cases such as $\rho=\{1,45\}$. For $\rho=1$, we can naively choose $Z_{2}$ odd fields $U=V=210$ in Fig. 1. However, an unsafe operator $V(U) \Delta(16) \bar{\Delta}(\overline{16})$ appears again. For $\rho=45$, one may choose $U=45$ and $V=45$ or 54 , which is unfavored by an operator similar to $\rho=1$.

\section{CONCLUSION}

According to the observed Higgs mass at the LHC and the dark matter direct detection limits, the conventional MSSM - the simplest natural SUSY that is consistent with unification-is under more pressure than ever. Such stress can be greatly relaxed in the extended MSSM models such as the NMSSM and VMSSM, which retain the unification and are still simple. In this paper, following the assumptions that $W_{Y}$ is fixed by the SM matter content and its tera electron volt-scale extension and that they receive their masses from $W_{\mathrm{SB}}$ through the Higgs mechanism, we have studied the embeddings of these three models into SUSY GUTs.

First of all, we discussed the MSSM, in which the realistic $\mathrm{SU}(5)$ and $\mathrm{SO}(10)$ realizations serve as benchmark solutions to the SM flavor issue and neutrino masses. Then, we utilized the benchmark MSSM as guidance for the embedding of the NMSSM and VMSSM. We found that the embedding of the NMSSM is not viable due to a large amount of mixings between the singlet $N$ and the Higgs fields responsible for the GUT symmetry breaking. But the problem can be evaded in the VMSSM because the Higgs field $\rho$ that provides 16-dimensional VL mass of order teraelectron-volt scale can avoid the same problems the singlet $N$ encounters due to the intermediate mass scale in the 126dimensional Higgs.

\section{ACKNOWLEDGMENTS}

The author thanks the anonymous referee for useful suggestions. This work is supported by the National Natural Science Foundation of China under Grant No. 11775039 and the Fundamental Research Funds for the Central Universities with Project No. cqu2017hbrc1B05 at Chongqing University.
[1] J. R. Ellis, S. Kelley, and D. V. Nanopoulos, Phys. Lett. B 260, 131 (1991).

[2] P. Langacker and M. x. Luo, Phys. Rev. D 44, 817 (1991).

[3] U. Amaldi, W. de Boer, and H. Furstenau, Phys. Lett. B 260, 447 (1991).

[4] C. Giunti, C. W. Kim, and U. W. Lee, Mod. Phys. Lett. A 06, 1745 (1991).

[5] G. Aad et al. (ATLAS Collaboration), Phys. Lett. B 716, 1 (2012).

[6] S. Chatrchyan et al. (CMS Collaboration), Phys. Lett. B 716, 30 (2012).
[7] M. Carena, S. Gori, N. R. Shah, and C. E. M. Wagner, J. High Energy Phys. 03 (2012) 014.

[8] L. J. Hall, D. Pinner, and J. T. Ruderman, J. High Energy Phys. 04 (2012) 131.

[9] J. J. Cao, Z. X. Heng, J. M. Yang, Y. M. Zhang, and J. Y. Zhu, J. High Energy Phys. 03 (2012) 086.

[10] H. Baer, V. Barger, and H. Serce, Phys. Rev. D 94, 115019 (2016).

[11] J. Cao, Y. He, L. Shang, W. Su, P. Wu, and Y. Zhang, J. High Energy Phys. 10 (2016) 136.

[12] P. Langacker, Phys. Rep. 72, 185 (1981). 
[13] H. Murayama and A. Pierce, Phys. Rev. D 65, 055009 (2002).

[14] J. Hisano, D. Kobayashi, T. Kuwahara, and N. Nagata, J. High Energy Phys. 07 (2013) 038.

[15] B. Bajc, S. Lavignac, and T. Mede, J. High Energy Phys. 01 (2016) 044.

[16] P. Fayet and S. Ferrara, Phys. Rep. 32, 249 (1977).

[17] K. S. Babu, I. Gogoladze, M. U. Rehman, and Q. Shafi, Phys. Rev. D 78, 055017 (2008).

[18] S. Zheng, Phys. Rev. D 98, 035028 (2018).

[19] S. Zheng, Eur. Phys. J. C 77, 588 (2017).

[20] H. Georgi and S. L. Glashow, Phys. Rev. Lett. 32, 438 (1974).

[21] H. Georgi, Particles and Fields 1974, edited by C. E. Carlson (AIP, New York, 1975), p. 575.

[22] H. Fritzsch and P. Minkowski, Ann. Phys. (N.Y.) 93, 193 (1975).

[23] S. Dimopoulos and H. Georgi, Nucl. Phys. B193, 150 (1981).

[24] N. Sakai, Z. Phys. C 11, 153 (1981).

[25] S. Dimopoulos, S. Raby, and F. Wilczek, Phys. Rev. D 24, 1681 (1981).

[26] G. Anderson, S. Dimopoulos, L. J. Hall, S. Raby, and G. D. Starkman, Phys. Rev. D 49, 3660 (1994).

[27] H. Georgi and C. Jarlskog, Phys. Lett. 86B, 297 (1979).

[28] H. Fritzsch, Nucl. Phys. B155, 189 (1979).

[29] P. Minkowski, Phys. Lett. B 67, 421 (1977).

[30] T. Yanagida, Conf. Proc. C 7902131, 95 (1979).

[31] M. Gell-Mann, P. Ramond, and R. Slansky, Conf. Proc. C 790927, 315 (1979).

[32] R. N. Mohapatra and G. Senjanovic, Phys. Rev. Lett. 44, 912 (1980).

[33] G. Altarelli, F. Feruglio, and I. Masina, J. High Energy Phys. 11 (2000) 040.
[34] H. Georgi and D. V. Nanopoulos, Nucl. Phys. B155, 52 (1979).

[35] H. Georgi and D. V. Nanopoulos, Nucl. Phys. B159, 16 (1979).

[36] K. S. Babu and R. N. Mohapatra, Phys. Rev. Lett. 70, 2845 (1993).

[37] J. Schechter and J. W. F. Valle, Phys. Rev. D 22, 2227 (1980).

[38] R. N. Mohapatra and G. Senjanovic, Phys. Rev. D 23, 165 (1981).

[39] G. Lazarides, Q. Shafi, and C. Wetterich, Nucl. Phys. B181, 287 (1981).

[40] T. Fukuyama and N. Okada, J. High Energy Phys. 11 (2002) 011.

[41] B. Bajc, G. Senjanovic, and F. Vissani, Phys. Rev. Lett. 90, 051802 (2003).

[42] K. S. Babu and C. Macesanu, Phys. Rev. D 72, 115003 (2005).

[43] A. Dueck and W. Rodejohann, J. High Energy Phys. 09 (2013) 024.

[44] T. Fukuyama, A. Ilakovac, T. Kikuchi, S. Meljanac, and N. Okada, Eur. Phys. J. C 42, 191 (2005).

[45] S. Bertolini, T. Schwetz, and M. Malinsky, Phys. Rev. D 73, 115012 (2006).

[46] B. Bajc, A. Melfo, G. Senjanovic, and F. Vissani, Phys. Lett. B 634, 272 (2006).

[47] C. S. Aulakh and S. K. Garg, Nucl. Phys. B757, 47 (2006).

[48] K. S. Babu, B. Bajc, and S. Saad, J. High Energy Phys. 10 (2018) 135.

[49] R. N. Mohapatra and M. Severson, J. High Energy Phys. 09 (2018) 119.

[50] T. Fukuyama, A. Ilakovac, T. Kikuchi, S. Meljanac, and N. Okada, J. Math. Phys. (N.Y.) 46, 033505 (2005). 